\title{
Spectroscopy and Femtosecond Dynamics of Type-II CdSe/ZnTe Core-Shell Semiconductor Synthesized via the CdO Precursor
}

\author{
Chun-Yen Chen, Chiu-Ting Cheng, Jen-Kan Yu, Shih-Chieh Pu, Yi-Ming Cheng, and \\ Pi-Tai Chou*
}

Department of Chemistry, National Taiwan University, Taipei, 106, Taiwan

\author{
Yi-Hsuan Chou and Hsin-Tien Chiu* \\ Department of Applied Chemistry, National Chiao Tung University, Hsinchu 300, Taiwan
}

Received: February 24, 2004; In Final Form: May 12, 2004

\begin{abstract}
$\mathrm{CdSe} / \mathrm{ZnTe}$ type-II quantum dots (QDs) synthesized via the $\mathrm{CdO}$ precursor are reported. Spectroscopic and femtosecond dynamic measurements reveal that the rate of photoinduced electron/hole spatial separation decreases with increases in the size of the core, and it is independent of the thickness of the shell in the $\mathrm{CdSe} / \mathrm{ZnTe} \mathrm{QDs}$. The results are consistent with the binding strength of the electron and hole confined at the center of CdSe. The correlation between the core/shell size and the electron/hole spatial separation rate resolved in this study may provide valuable information for applications where rapid photoinduced carrier separation followed by charge transfer into a matrix or electrode is crucial, such as in photovoltaic devices.
\end{abstract}

\section{Introduction}

In contrast to type-I core/shell semiconductor quantum dots (QDs), ${ }^{1-7}$ which possess band offsets in that the conduction band of the shell is higher in energy than that of the core, while the relative energy level is opposite in the valence band, typeII QDs have both valence and conduction bands in the core lower (or higher) than those in the shell materials. ${ }^{8}$ As a result, upon excitation, one carrier is predominantly confined to the core, while the other is confined to the shell. This spatial separation of charge carriers leads to several novel properties that differ intrinsically from those of the type-I QDs. On one hand, type-II structures allow reaching an interband emission with an energy gap that would be otherwise unavailable with type-I structures. On the other hand, the charge separation in type-II QDs should make these materials more suitable in, for example, photovoltaic applications, in which QDs may replace dye chromophores so that one of the photocarriers is injected from the QDs into a matrix before the occurrence of electron/ hole recombination.

Most type-II nanocomposites reported thus far have been grown by molecular beam epitaxy. ${ }^{9,10}$ Very recently, based on a colloidal template, an exquisite synthetic route on type-II $\mathrm{CdTe} / \mathrm{CdSe}$ (core/shell) and CdSe/ZnTe (core/shell) QDs was achieved. ${ }^{8}$ This soft-template approach is remarkable in that it offers both feasibility and versatility toward further chemical modification. As a result, the associated emission properties have been systematically analyzed in $\mathrm{CdTe} / \mathrm{CdSe}$, while the corresponding data on $\mathrm{CdSe} / \mathrm{ZnTe}$ is obscure, possibly due to its weak interband emission at the near-IR (NIR) region. ${ }^{8}$ In contrast to thorough investigations of the electron/hole relaxation pathways on the type-I nanoparticles, ${ }^{11-20}$ to our knowledge, dynamics of interfacial electron/hole separation for these type-II QDs have

* Corresponding authors: (P.-T.C.) chop@ntu.edu.tw; (H.-T.C.) htchiu@ cc.nctu.edu.tw. not yet been systematically studied. We herein present the spectroscopy and femtosecond upconversion approaches for type-II QDs, CdSe/ZnTe (core/shell), which was synthesized via an alternative synthetic route incorporating the greener and safer $\mathrm{CdO}$ (core), sulfur powder (shell), and zinc stearate (shell) as precursors instead of $\mathrm{Cd}(\mathrm{Me})_{2}$ and $(\mathrm{TMS})_{2} \mathrm{Se}^{8}$

\section{Experimental Section}

Synthesis. Tri- $n$-octylphosphine oxide (TOPO, 99\%), tri- $n$ butylphosphine (TBP, technical grade $98 \%$ ) and hexadecylamine (HDA, 90\%) were purchased from Aldrich, SHOWA and TCI, respectively. CdO (99.99\%), selenium (Se) powder 200 mesh $(>99.5 \%)$, sulfur $(\mathrm{S})$ powder $(99.5 \%)$, and tellurium $(\mathrm{Te})$ powder 200 mesh $(99.8 \%)$ were obtained from Strem, ACROS, Alfa Aesar, and Aldrich, respectively. Zinc stearate was purchased from Riedel-deHaën.

The preparation of the $\mathrm{CdSe}$ core from $\mathrm{CdO}$ was done according to a method previously reported by Peng and coworkers $^{21}$ with a slight modification. Briefly, a Se injection solution containing $0.079 \mathrm{~g}$ of $\mathrm{Se}(1 \mathrm{mmol})$ was prepared in a glovebox by dissolving Se powder in $0.3 \mathrm{~mL}$ of tri- $n$ butylphosphine and then diluted with $1.681 \mathrm{~g}$ di-n-octylamine. The Se injection solution was then brought out of the glovebox in a vial sealed with a rubber subseal. A mixture of $\mathrm{CdO}(0.0128$ $\mathrm{g}, 0.10 \mathrm{mmol})$ and stearic acid $(0.114 \mathrm{~g}, 0.40 \mathrm{mmol})$ was heated in a three-neck flask to $140{ }^{\circ} \mathrm{C}$ under Ar flow. After $\mathrm{CdO}$ was completely dissolved, the mixture was allowed to cool to room temperature. $1.94 \mathrm{~g}$ of the TOPO and HDA was then added to the flask, and the mixture was heated to $320{ }^{\circ} \mathrm{C}$. At this temperature, the Se injection solution was quickly injected into the hot solution. The reaction mixture was allowed to cool to $290{ }^{\circ} \mathrm{C}$ for the growth of the CdSe nanocrystals. Various sizes of CdSe QDs were obtained by the time period required to terminate the reaction upon cooling. CdSe QDs were then precipitated out from the growth solution by adding methanol. 
For a typical protocol used to obtain the CdSe/ZnTe core/ shell nanoparticles, the precipitated CdSe QDs $(0.020 \mathrm{~g})$ were dispersed in TOPO (2.20 g) and HDA (1.26 g) then heated to $190{ }^{\circ} \mathrm{C}$. On the other hand, zinc stearate $(0.316 \mathrm{~g})$ was dissolved in $2.5 \mathrm{~mL}$ of TBP upon gentle heating (ca. $80^{\circ} \mathrm{C}$ ). After cooling to room temperature, the resulting $0.2 \mathrm{M}$ solution was mixed with $2.5 \mathrm{~mL}$ of a $0.2 \mathrm{M}$ solution of Te in TBP. Using a syringe pump, this mixture was injected within $1 \mathrm{~h}$ into the reaction flask containing the core nanocrystals at $190-200{ }^{\circ} \mathrm{C}$. After the addition was completed the crystals were annealed at 190 ${ }^{\circ} \mathrm{C}$ for an additional $1-1.5 \mathrm{~h}$. Various sizes of core/shell nanoparticles were obtained by adjusting the concentrations of zinc stearate and Te in TBP as well as the corresponding injection period.

Measurements. The shapes of QDs were obtained with a Hitachi H-7100 transmission electron microscope (TEM). UVvis steady-state absorption and emission spectra were recorded by a Hitachi (U-3310) spectrophotometer and an Edinburgh (FS920) fluorimeter, respectively. The type-II NIR emission spectra were obtained by exciting the sample solution under a front-face excitation configuration using Ar ion laser (488 or $514 \mathrm{~nm}$, Coherent Innova 90). The emission was then sent through an NIR-configured Fourier transform interferometer (Bruker Equinox 55) and detected by a red sensitive photomutiplier (Hamamatsu model R5509-72) operated at $-80{ }^{\circ} \mathrm{C}$. Typically, the integration time for each measurement was about $15 \mathrm{~min}$. Solvents such as toluene and tetrahydrofuran (THF) were of spectragrade quality (Merck Inc.) and were used right after being received.

Nanosecond lifetime studies were performed by an Edinburgh FL 900 photon-counting system with a hydrogen-filled/or a nitrogen lamp as the excitation source. The emission decays were analyzed by the sum of exponential functions, which allows partial removal of the instrument time broadening and consequently renders a temporal resolution of $\sim 200 \mathrm{ps}$. The fluorescence dynamics in the pico-femtosecond region were measured using a femtosecond fluorescence upconversion system (FOG100, CDP Lasers \& Scanning Systems). Briefly, the fundamental of a Kerr lens mode-locked titanium (Ti): sapphire laser (Tsunami, Spectra-Physics) pumped by an argon laser (BeamLok 2060, Spectra-Physics) at 770-810 nm with an average power of $800 \mathrm{~mW}$ and a repetition rate of $82 \mathrm{MHz}$ was used as the light source. About $500 \mathrm{~mW}$ of this output was frequency-doubled in a $0.5 \mathrm{~mm}$ BBO crystal. The frequencydoubled pulses were focused on the sample located in a rotating cell. The fluorescence was collected with an achromatic doublet. The unconverted pulses were sent along an optical delay line before being focused together with the fluorescence on a 0.5 $\mathrm{mm}$ BBO crystal. The polarization of the pump pulses was at the magic angle $\left(54.7^{\circ}\right)$ with respect to that of the gate pulses at $800 \mathrm{~nm}$ to eliminate the fluorescence anisotropy. The upconverted signal was then sent into a spectrograph, and its intensity was measured with a photomultiplier tube operated at the photon counting mode. The full width at half-maximum (fwhm) of the response function of the setup, measured by autocorrelation, was about $150 \mathrm{fs}$.

\section{Results and Discussion}

Figure 1 depicts TEM images of various sizes of CdSe/ZnTe type-II QDs and their initial core CdSe particles. The absorption and emission spectra of the CdSe cores and the corresponding CdSe/ZnTe type-II QDs are shown in Figure 2 and Figure 3, respectively, in which the core (core/shell) sizes, measured by TEM image, were calculated to be 3.5 (4.3), 4.1 (5.0), and 5.5
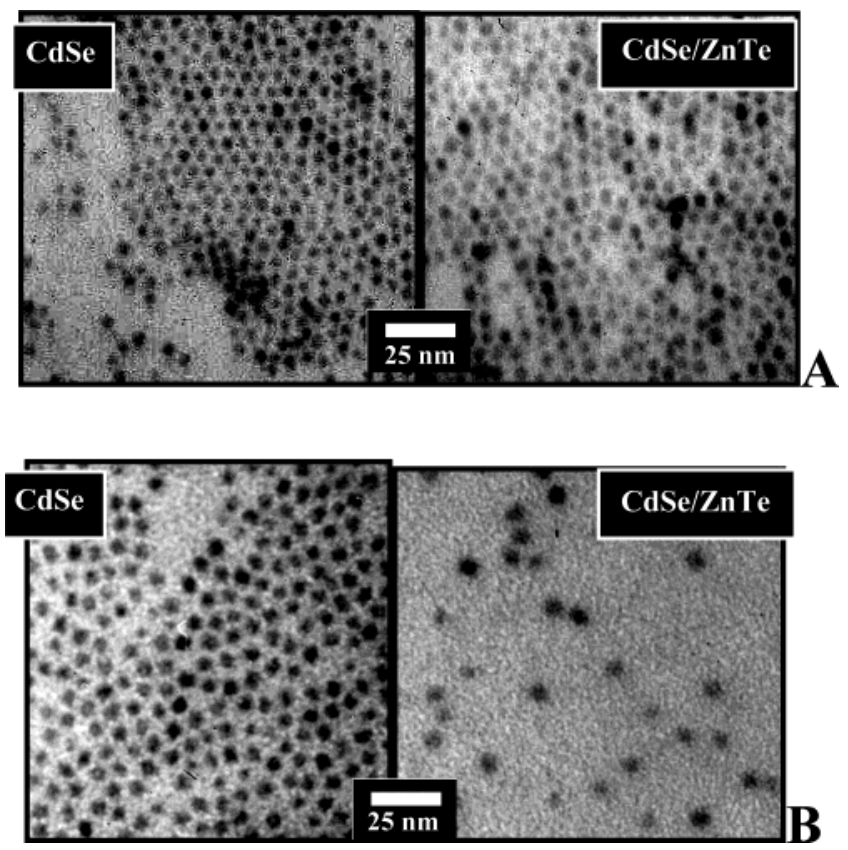

Figure 1. TEM images of the samples with an average of (A) $3.5 \mathrm{~nm}$ diameter CdSe QDs (left) and 4.3 nm CdSe/ZnTe QDs (right), (B) 5.5 $\mathrm{nm}$ diameter CdSe QDs (left) and $6.3 \mathrm{~nm} \mathrm{CdSe} / \mathrm{ZnTe}$ QDs (right).

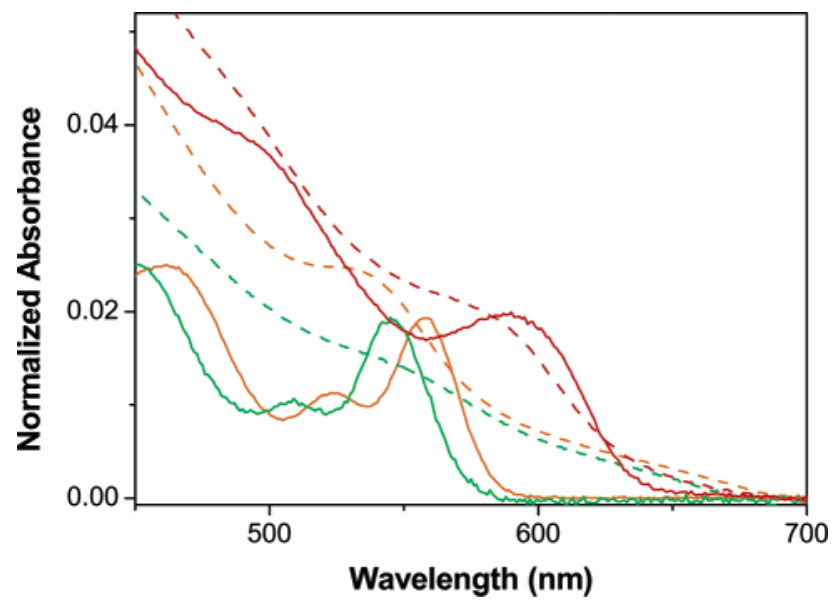

Figure 2. Absorption spectra of CdSe core $(-)$ and CdSe/ZnTe coreshell (- - ) QDs in toluene by varying the core size from $3.5 \mathrm{~nm}$ (green) and $4.1 \mathrm{~nm}$ (orange) to $5.5 \mathrm{~nm}$ (red). The thickness of the shell is $\sim 0.8 \pm 0.1 \mathrm{~nm}$ for all three cases.

(6.3) $\mathrm{nm}$ in average. Since CdSe/ZnTe QDs were prepared from the same batch of the CdSe precursor, the ZnTe thickness of three samples was derived to be $0.8,0.9$, and $0.8 \mathrm{~nm}$, which, within experimental error, could be treated as a constant value. Thus, as shown in Figure 3, similar to that of core CdSe, the NIR emission band for CdSe/ZnTe QDs also revealed systematic red shift upon increasing the size of the CdSe core. The results, in combination with a rather narrow fwhm of $\sim 1000-1300$ $\mathrm{cm}^{-1}$, rule out the possibility that the NIR emission bands result from a "deep trap" luminescence often observed in cores. In addition, a point worthy of note is that the synthetic route based on the $\mathrm{CdO}$ precursor resulted in a predominant $\mathrm{CdSe}$ core emission free of the deep-trap interference in this study. Thus, alternatively, it is more plausible to ascribe the NIR emission band originating from the radiative recombination of the electron-hole pair across the core-shell interface, i.e., the typeII interband emission. ${ }^{8}$ Theoretically, the energy gap of the interband emission correlates with the band offsets of the materials composing the core and the shell. If core/shell 


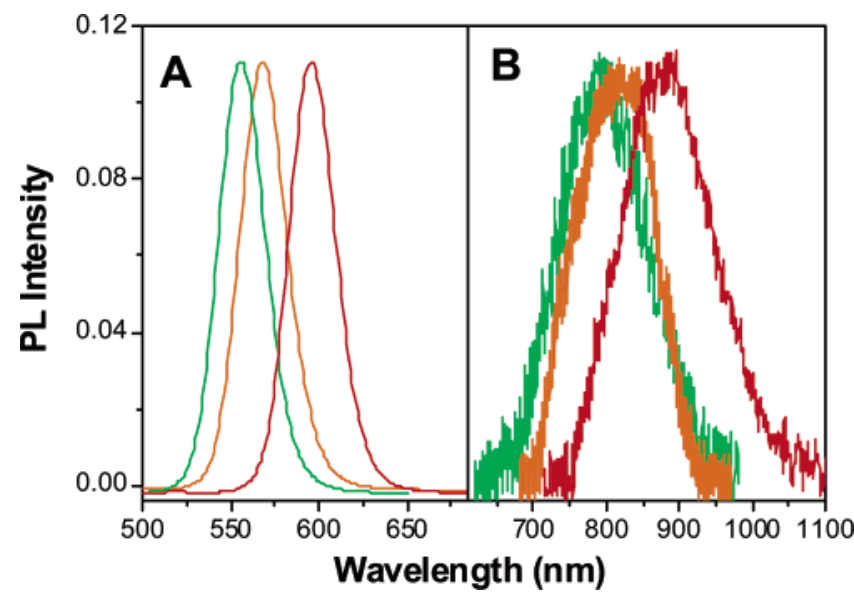

Figure 3. (A) Emission spectra of CdSe core QDs and (B) CdSe/ZnTe core-shell QDs in toluene by varying the core size from 3.5 and 4.1 to $5.5 \mathrm{~nm}$ (from left to right). The thickness of the shell is $\sim 0.8 \pm 0.1$ $\mathrm{nm}$ for all three cases. $\lambda_{\mathrm{ex}}$ : $488 \mathrm{~nm}$.

TABLE 1: Room-Temperature Luminescence Decays for Various Sizes of CdSe and Corresponding CdSe/ZnTe QDs in Toluene

\begin{tabular}{|c|c|c|c|c|c|c|c|}
\hline \multicolumn{4}{|c|}{ CdSe core } & \multicolumn{4}{|c|}{$\mathrm{CdSe} / \mathrm{ZnTe}$ core - shell } \\
\hline $\begin{array}{l}\text { size } \\
(\mathrm{nm})\end{array}$ & $\begin{array}{c}\mathrm{PL} \\
\left(\lambda_{\max }\right)\end{array}$ & $\begin{array}{c}\tau_{\mathrm{f}}^{b} \\
(\mathrm{~ns})\end{array}$ & QY & $\begin{array}{l}\operatorname{size}^{a} \\
(\mathrm{~nm})\end{array}$ & $\begin{array}{c}\mathrm{PL} \\
\left(\lambda_{\max }\right)\end{array}$ & $\begin{array}{c}\tau_{\mathrm{f}^{c}} \\
(\mathrm{~ns})\end{array}$ & $\begin{array}{c}\text { QY } \\
\left(\times 10^{3}\right)\end{array}$ \\
\hline 3.5 & 555 & & $0_{5}$ & & 790 & 55.7 & \\
\hline 4.1 & 570 & & & & 81 & 52.4 & \\
\hline 5.5 & 610 & 19.9 & 0.56 & 6.3 & 875 & 53.8 & 3.81 \\
\hline
\end{tabular}

${ }^{a}$ The core-shell size was synthesized from its corresponding core, and the average shell thickness was $\sim 0.8 \mathrm{~nm} .{ }^{b}$ The average decay time at room temperature. ${ }^{c} \tau_{\mathrm{f}}$ was measured at peak of the interband emission.

materials remain unchanged, the interband exciton emission should also be dependent on the size of the core and/or the thickness of the shell. This is clearly demonstrated in Figure 3. Upon the increase in the size of CdSe cores from 3.5 to 5.5 $\mathrm{nm}$, as indicated by the shift of the emission peak wavelength from 550 to $610 \mathrm{~nm}$, the emission of CdSe/ZnTe, coated with a similar thickness $(\sim 0.8 \mathrm{~nm})$ of shell materials (ZnTe), shows a systematically bathochromic shift from 790 (core $3.5 \mathrm{~nm} / \mathrm{shell}$ $0.8 \mathrm{~nm}$ ) to $875 \mathrm{~nm}$ (core $5.5 \mathrm{~nm} /$ shell $0.8 \mathrm{~nm}$ ). The results can be rationalized by lowering the $\mathrm{CdSe}$ conduction band at the edge, resulting in the decrease between the CdSe (higher band) and ZnTe (lower band) band gap.

Table 1 lists room-temperature luminescence decays for various sizes of CdSe and the corresponding CdSe/ZnTe QDs. The average population decay times for the core-only CdSe consistently decrease from 64 to $20 \mathrm{~ns}$ as the size of the core increases from 3.5 to $5.5 \mathrm{~nm}$. Conversely, the average decay times of CdSe/ZnTe QDs, under the same shell thickness of $\sim 0.8 \mathrm{~nm}$, are about $50-55 \mathrm{~ns}$ with a lack of correlation with respect to the particle size. In contrast to the strong visible emission of the core $\left(\Phi_{\mathrm{f}}>0.5\right.$, see Table 1$)$, the photoemission from CdSe/ZnTe QDs was observed in the NIR region with a relatively much weaker intensity. Comparing the emission intensity with that of meso-tetraphenylporphyrin $\left(\Phi_{\mathrm{f}} \sim 0.11\right)$ in toluene, ${ }^{22}$ the quantum efficiency of the NIR interband emission was calculated to be on the order of $10^{-3}$ (see Table 1). The low emission yield, along with slow nonradiative processes $(\sim 50 \mathrm{~ns})$, renders a radiative decay rate constant in the range of $10^{4}-10^{5} \mathrm{~s}^{-1}$, which is much smaller than that of the core-only CdSe QDs by approximately 3 orders of magnitude (see Table 1). The extraordinarily long radiative lifetime

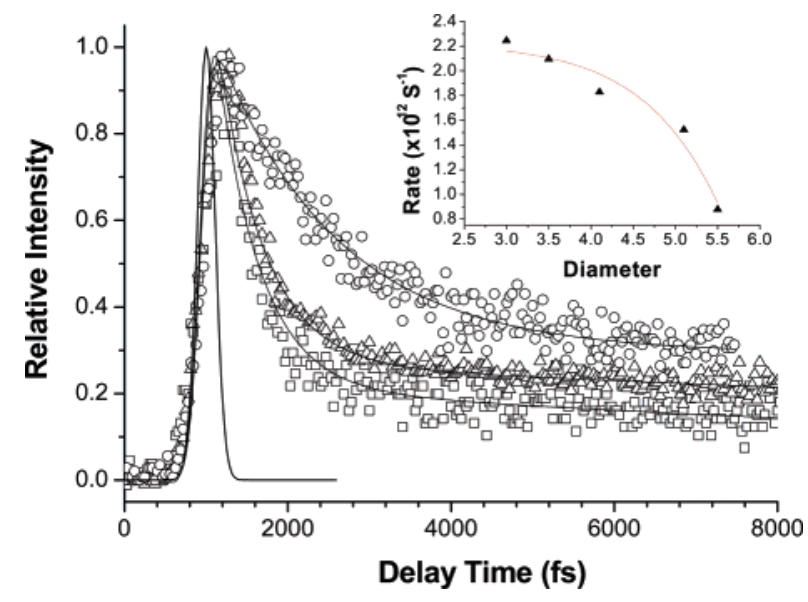

Figure 4. Fluorescence upconversion signal for CdSe/ZnTe QDs in toluene with a core diameter of $(\square) 3.5 \mathrm{~nm}(555 \mathrm{~nm}),(\triangle) 4.1 \mathrm{~nm}$, and (○) $5.5 \mathrm{~nm}$. (一) system response function. Emission is monitored at the peak wavelength of the corresponding core. Inset: The plot of electron-hole interfacial separation rate as a function of the core diameter. The thickness of shell (ZnTe) is $\sim 0.8 \mathrm{~nm}$ for all QDs. $\lambda_{\text {ex }}$ : $375 \mathrm{~nm}$.

can be rationalized by the slow, spatially separated electronhole recombination of the excitons in type-II structures.

Femtosecond dynamics were performed to gain further insights into the dynamics of photoinduced electron-hole separation in the early stage. On the basis of the fluorescence upconversion technique, attempts to acquire the rise dynamics of the interband emission ( $>700 \mathrm{~nm}$ ) unfortunately failed mainly due to the exceedingly small radiative decay rate (vide supra). Alternatively, we have monitored the signal at the region of 550-630 nm supposed to be the precursor core emission, which, due to the dominant electron/hole pair spatially separated process, is negligibly small in the steady state intensity for the $\mathrm{CdSe} / \mathrm{ZnTe}$ QDs. Figure 4 reveals the decay dynamics for various sizes of $\mathrm{CdSe} / \mathrm{ZnTe} \mathrm{QDs}$ monitored at the peak wavelength of the corresponding core CdSe QDs. It turned out that the experimental results could be qualitatively fitted well by two components: (1) a very fast but resolvable decay component, of which the lifetime is within several hundred femtoseconds, and (2) a much slower component, of which the amplitude can be treated as a constant throughput the measured range of $10 \mathrm{ps}$. Component 2 was further resolved to be around $7-8 \mathrm{~ns}$ by the time-correlated single photon counting experiment.

Because the excitation wavelength was tuned at 375-400 $\mathrm{nm}$ in the fluorescence upconversion study, we realized that direct excitation of the ZnTe shell was also possible. ${ }^{23,24}$ Support of this viewpoint was rendered by both steady-state and nanosecond time-correlated single photon counting measurements shown in the insert of Figure 5, in which a weak emission with the peak wavelength of $\sim 450 \mathrm{~nm}\left(\tau_{\mathrm{f}} \sim 7 \mathrm{~ns}\right)$ was resolved upon excitation at $<400 \mathrm{~nm}$. In comparison, the $450 \mathrm{~nm}$ band was absent in the CdSe core QDs. Accordingly, the constant value shown in the upconversion experiment is most plausibly attributed to the directly excited $\mathrm{ZnTe}$ decay component. However, for the type-II CdSe/ZnTe QDs, the photoexcitation of the shell should result in a rapid electron transfer due to the same charge separated state as the core excitation. It is thus more likely to attribute the slow decay component to a small concentration of free-standing $\mathrm{ZnTe}$ particles, resulting from nucleation during the shell growth (vide infra).

As to the fast decay component, when the size of the CdSe core increased to $3.5,4.1$, and $5.5 \mathrm{~nm}$, the rate of the decay 


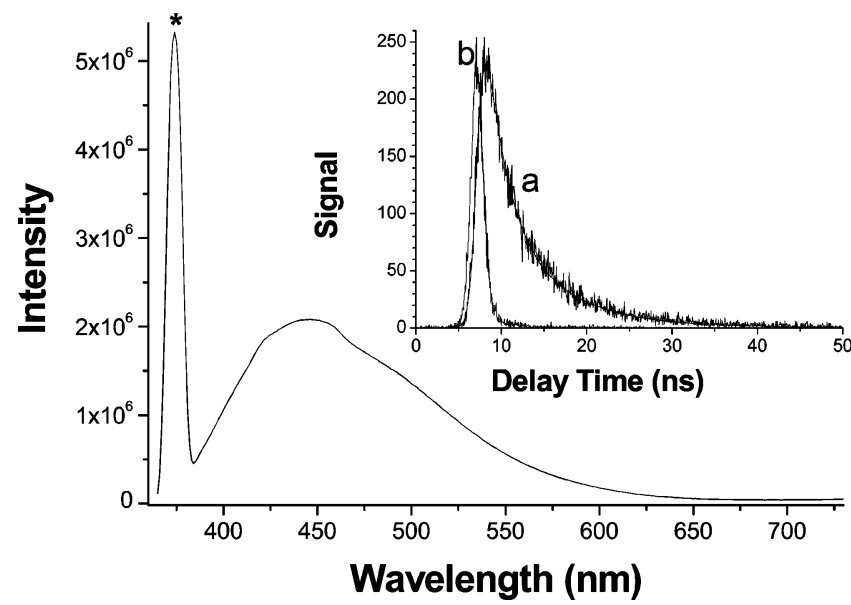

Figure 5. Steady-state emission of CdSe/ZnTe core-shell QDs (3.5/0.8 $\mathrm{nm}$ ) excited at $375 \mathrm{~nm}$. $*$ denotes Rayleigh scattering. Inset: (a) decay dynamics monitored at $450 \mathrm{~nm}\left(\tau_{\mathrm{f}} \sim 7 \mathrm{~ns}, \lambda_{\mathrm{ex}}=375 \mathrm{~nm}\right)$; (b) system response function.

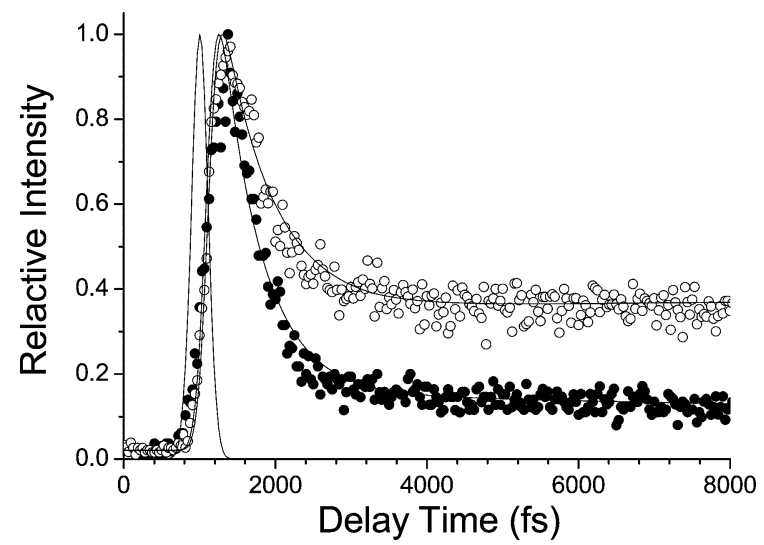

Figure 6. Same size of CdSe core $(\sim 5.1 \mathrm{~nm})$ coated with $(\bullet) 0.8$ and (O) $1.8 \mathrm{~nm}$ thickness of the ZnTe shell. (-) system response function. $\lambda_{\text {ex }}: 375 \mathrm{~nm}$.

component (1) systematically decreased to $477 \pm 50,547 \pm 55$, and $1140 \pm 70 \mathrm{fs}^{-1}$, respectively. Assuming that the fast quenching of the core emission in $\mathrm{CdSe} / \mathrm{ZnTe}$ is dominated by the rate of electron/hole spatial separation, the plot for the rate of interfacial hole-transfer vs the core diameter reveals a convexcurve-like correlation (see the insert of Figure 4). In contrast, a nearly shell-thickness-independent rate of electron/hole spatial separation was observed. For example, by fixing the CdSe size at $5.1 \mathrm{~nm}$, while the $\mathrm{ZnTe}$ thickness varied from 0.8 to $1.8 \mathrm{~nm}$, the rates of fast decay component were measured to be $657 \pm 50$ and $672 \pm 60 \mathrm{fs}^{-1}$, respectively (see Figure 6), which, within experimental error, are identical. Note that in this case the NIR emission red shifts from 820 (core $5.1 \mathrm{~nm} /$ shell $0.8 \mathrm{~nm}$ ) to 832 $\mathrm{nm}$ (core $5.1 \mathrm{~nm} /$ shell $1.8 \mathrm{~nm}$, not shown here) possibly due to the increase of the $\mathrm{ZnTe}$ valence band at the edge. Furthermore, as shown in Figure 6, the intensity of the long decay component ( $\tau_{\mathrm{f}} \sim 7 \mathrm{~ns}$ ) increases by $\sim 2$-fold upon increasing the ZnTe thickness from 0.8 to $1.8 \mathrm{~nm}$. Since the increase of shell thickness requires a longer nucleation time for $\mathrm{ZnTe}$, the freestanding ZnTe is expected to increase as well, providing further support for the assignment of the $450 \mathrm{~nm}$ band to the ZnTe emission.

The above results, on one hand, may be qualitatively rationalized in terms of a Coulombic interaction between the electrons and the holes that participate in the interband transition. If one assumes the quenching of the core emission in type-II QDs to be dominated by the hole tunneling process, in a one- dimensional approach (along the radius $z$ direction), the holetransfer rate $k_{\mathrm{t}}$ can be expressed as

$$
k_{t} \propto \exp \left[-\int \sqrt{\frac{2 m V(z)}{\hbar}} \mathrm{d} z\right]
$$

where $V(z)$ denotes the Coulomb barrier for a hole confined in the local potential minima at the edge of CdSe. Theoretical approaches based on the variation method have shown that both height and width of $V(z)$ are dependent on the size of the core $(\mathrm{CdSe}){ }^{23,24}$ Electrons that are strongly confined in the CdSe layer create Coulomb wells at the center of the CdSe valence barriers. Thus, with larger sizes of CdSe QDs, the local potential minimum can confine the majority of photogenerated holes so that the hole-transfer into the ZnTe becomes more difficult. Unfortunately, the self-consistent approach for the potential energy surface of $V(z)$ cannot be solved exactly in eq 1 , so that its application to fit the experimental results is infeasible. Nevertheless, this simple approach qualitatively predicts a coreradius dependent hole-tunneling rate. On the other hand, it is also possible that the finite rate of charge separation is due to the low electron-phonon coupling, causing there a too small coupling between the initial and charge-separated states. Thus, a mechanism incorporating energy dissipation or coupling bottleneck ${ }^{25,26}$ might be more plausible. Nevertheless, due to the lack of a fundamental basis, the actual mechanism of relaxation dynamics remains unsolved, which should bring up a broad spectrum of interest in both experimental and theoretical approaches in the future.

In conclusion, we have prepared CdSe/ZnTe QDs from the greener $\mathrm{CdO}$ precursor to perform spectroscopy and dynamics of the type-II interband emission. The hole transfer rate as a function of the core/shell size has been systematically approached. The results indicate that the rate of hole transfer decreases with increasing the size of the cores and is independent of shell-thickness in the CdSe/ZnTe QDs. This degree of control can be especially crucial in applications where rapid carrier separation followed by charge transfer into a matrix or electrode is important, as in photovoltaic devices.

Acknowledgment. We are grateful to the CTCI Foundation and National Science Council, Taiwan, for the financial support. We also thank Ching-Yen Lin and Chih-Yuan Tang in the Instrumentation Center, National Taiwan University, for assistance with the TEM measurements.

\section{References and Notes}

(1) Brus, L. E. J. Phys. Chem. 1986, 90, 2555

(2) Alivisatos, A. P. Science 1996, 271, 933.

(3) Danek, M.; Jensen, K. F.; Murray, C. B.; Bawendi, M. G. Chem. Mater. 1996, 8, 173.

(4) Nirmal, M.; Brus, L. E. Acc. Chem. Res. 1999, 32, 407.

(5) Mattoussi, H.; Mauro, J. M.; Goodman, E.; Anderson, G. P.; Sundar, V. C.; Mikulec, F. V.; Bawendi, M. G. J. Am. Chem. Soc. 2000, 122, 12142

(6) Talapin, D. V.; Rogach, A. L.; Kornowski, A.; Haase, M.; Weller, H. Nano Lett. 2001, 1, 207.

(7) Reiss, P.; Bleuse, J.; Pron, A. Nano Lett. 2002, $2,781$.

(8) Kim, S.; Fisher, B.; Eisler, H. J.; Bawendi, M. G. J. Am. Chem. Soc. 2003, 125, 11466.

(9) Hatami, F.; Grundmann, M.; Ledentsov, N. N.; Heinrichsdorff, F.; Heitz, R.; Böhrer, J.; Bimberg, D.; Ruvimov, S. S.; Werner, P.; Ustinov, V. M.; Kop'ev, P. S.; Alferov, Z. I. Phys. Rev. B 1998, 57, 4635.

(10) Youn, H. C.; Baral, S.; Fendler, J. H. J. Phys. Chem. 1988, 92, 6320.

(11) Chikan, V.; Kelley, D. F. Nano Lett. 2002, 2, 141.

(12) Chikan, V.; Kelley, D. F. Nano Lett. 2002, 2, 1015.

(13) Tu, H.; Chikan, V.; Kelley, D. F. J. Phys. Chem. B 2003, 107, 10389. 
(14) Woggon, U.; Geissen, H.; Gindele, F.; Wind, O.; Fluegel, B.; Peyghambarian, N. Phys. Rev. B 1996, 54, 17681

(15) Klimov, V. I.; McBranch, D. W. Phys. Rev. Lett. 1998, 80, 4028.

(16) Guyot-Sionnest, P.; Shim, M.; Matranga, C.; Hines, M. Phys. Rev. $B$ 1999, 60, R2181.

(17) Klimov, V. I.; Schwarz, C. J.; McBranch, D. W.; Leatherdale, C. A.; Bawendi, M. G. Phys. Rev. B 1999, 60, R2177.

(18) Klimov, V. I.; Milhailosky, A. A.; McBranch, D. W.; Leatherdale, C. A.; Bawendi, M. G. Phys. Rev. B 2000, 61, R13349.

(19) Underwood, D. F.; Kippeny, T.; Rosenthal, S. J. J. Phys. Chem. B 2001, 105, 436 .
(20) Burda, C.; Link, S.; Mohamed, M.; El-Sayed, M. A. J. Phys. Chem $B$ 2001, 105, 12286.

(21) Qu, L.; Peng, X. J. Am. Chem. Soc. 2002, 124, 2049.

(22) Seybold, P. G.; Gouterman, M. J. Mol. Spectrosc. 1969, 31, 1

(23) Lee, E. H.; Stoltz, S.; Chang, H. C.; Na, M. H.; Luo, H.; Petrou, A. Solid State Commun. 1998, 107, 177.

(24) Haetty, J.; Lee, E. H.; Luo, H.; Petrou, A.; Warnock, J. Solid State Commun. 1998, 108, 205.

(25) Bockelmann, U.; Bastard, G. Phys. Rev. B 1990, 42, 8947.

(26) Benisty, H.; Sotomayor-Torres, C.; Weisbuch, C. Phys. Rev. B 1991 